Rhode Island College

Digital Commons @ RIC

$5-1-2013$

\title{
Booking the Appointment: A Strategy to Reduce Readmissions in Heart Failure Patients
}

Lindsey Moran

Rhode Island College

Follow this and additional works at: https://digitalcommons.ric.edu/etd

Part of the Nursing Commons

\section{Recommended Citation}

Moran, Lindsey, "Booking the Appointment: A Strategy to Reduce Readmissions in Heart Failure Patients" (2013). Master's Theses, Dissertations, Graduate Research and Major Papers Overview. 220.

https://digitalcommons.ric.edu/etd/220

This Major Paper is brought to you for free and open access by the Master's Theses, Dissertations, Graduate Research and Major Papers at Digital Commons @ RIC. It has been accepted for inclusion in Master's Theses, Dissertations, Graduate Research and Major Papers Overview by an authorized administrator of Digital Commons @ RIC. For more information, please contact digitalcommons@ric.edu. 


\title{
BOOKING \\ THE APPOINTMENT: \\ A STRATEGY TO REDUCE \\ READMISSIONS IN HEART FAILURE PATIENTS
}

\author{
by \\ Lindsey Moran \\ A Major Paper Submitted in Partial Fulfillment \\ of the Requirements for the Degree of \\ Master of Science in Nursing \\ in \\ The School of Nursing \\ Rhode Island College
}

2013 


\begin{abstract}
Heart failure (HF) is a chronic condition that is the cause for many hospitalizations in the United States. Hospital readmission is a common problem in many chronic conditions, especially heart failure. The purpose of this research was to determine if scheduling a follow-up appointment with a primary care provider (PCP) or cardiologist prior to hospital discharge decreases 30 -day readmission rates in patients with a primary diagnosis of HF. A quasi-experimental, two-group study was performed at The Miriam Hospital, a 247-bed acute care hospital, with a sample of 60 patients. A retrospective chart audit was performed to determine if 30-day readmission rates were lower in those HF patients who had a follow-up appointment booked prior to hospital discharge than those who did not have the appointment booked. Charts were reviewed for patients discharged during June, July, August and September 2012. Basic descriptive statistics were performed as well as differences between groups. Thirty-day readmission rates were lower for those who had a follow-up appointment booked (22.58\%) compared those who did not have an appointment booked (31.03\%). The 30-day readmission rate for those who had an appointment with a PCP was higher than those who followed up with a cardiologist (33.33\% versus $7.69 \%$ ). These findings suggest that booking a follow-up appointment for HF patients with a cardiologist prior to hospital discharge may help to decrease 30-day readmission rates. This simple intervention can be performed by nonclinical, administrative staff and could save hospitals money if even one HF readmission were prevented.
\end{abstract}

Keywords: heart failure, readmission, follow-up 
Table of Contents

Table of Contents..................................................... page

Background and Statement of the Problem......................................

Review of the Literature ..................................................4

Theoretical Framework...........................................................

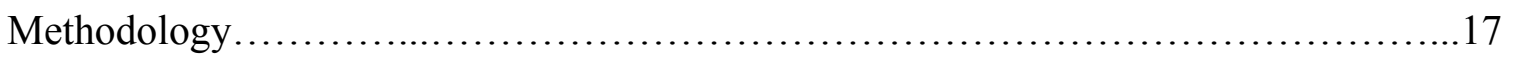

Results.................................................................. 20

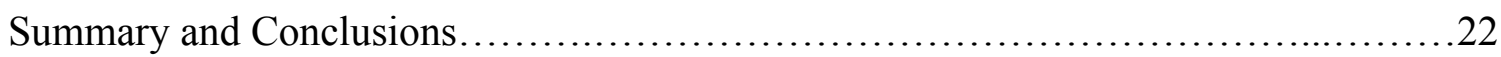

Recommendations and Implications.......................................... 26

References..............................................................29

Appendix................................................................. 32 
Booking the Appointment:

A Strategy to Reduce Readmissions in Heart Failure Patients

Background and Statement of the Problem

Heart failure (HF) is a chronic condition that occurs when the heart cannot pump enough blood and oxygen to other organs. As people are now living longer lives, many must deal with chronic conditions as they age. According to the Center for Disease Control (CDC, 2012), of the approximately 5.8 million people in the United States (US) living with $\mathrm{HF}$ one in five will die within one year of initial diagnosis. The most common causes of HF are coronary artery disease, high blood pressure and diabetes. Due to the large number of people living with HF in the US, management and treatment of this chronic condition has become quite costly. In 2010 the US spent approximately 39.2 billion dollars on HF (CDC, 2012). Hospital admissions and readmissions related to HF are major contributors to the high cost of treatment.

In $2005,17.6 \%$ of all hospital admissions resulted in readmissions within 30 days of discharge. Many of these readmissions may have been preventable. In general, those readmitted to the hospital are older adults with a variety of chronic conditions such as HF (Stone \& Hoffman, 2010). Of all medical and surgical diagnoses in Medicare beneficiaries, HF has the highest 30-day hospital readmission rate at $26.9 \%$ while the average readmission rate for all discharged patients was only $19.6 \%$. While patients with HF can be readmitted for a variety of reasons, it is noteworthy that $37.0 \%$ of readmissions are for HF itself (Jencks, Williams, \& Coleman, 2009). These findings suggest that HF readmissions are fairly predictable in patients who were initially admitted 
for HF. Given the high cost associated with hospital admissions and readmissions, Congress and President Obama identified reducing hospital readmissions as a way to decrease Medicare spending. Policy makers and researchers argue that while some hospital readmissions are appropriate, decreasing these rates will help to contain Medicare spending and improve the quality of care received by Medicare beneficiaries (Stone \& Hoffman, 2010).

Hospital readmission rates are not only being scrutinized by the federal government, but also by the general public. The Centers for Medicare and Medicaid Services (CMS) have recently made publicly available the 30-day readmission rates for hospitals nationwide on their website, Hospital Compare. On the Hospital Compare website, 30-day readmission rates for heart attack, HF, and pneumonia are compared to the national average (Stone \& Hoffman). This public reporting places great pressure on hospitals nationwide to create innovative ways to decrease their 30-day readmission rates, as it can be expected that savvy healthcare consumers will take this information into consideration when choosing which hospital they will receive care at.

Although not all readmissions are preventable, researchers feel that many could be prevented if the quality of care were improved in the following areas: Medicarecovered hospital stays; hospital discharge processes; and during follow-up after hospital discharge. Researchers have found several factors that contribute to hospital readmission overall including: inadequate follow-up care; poor patient compliance; inadequate relay of medical information from hospital providers to patients, caregivers and post-acute and 
long-term care providers; insufficient use of family caregivers; variation in hospital bed supply; medical errors; and deterioration (Stone \& Hoffman).

In summary, HF is a common chronic condition that often results in hospital admissions and readmissions, which are extremely costly. There is a need to identify strategies that are effective in decreasing readmission rates in patients with HF in order to provide higher quality of care, improve quality of life and decrease healthcare costs. The purpose of this project was to determine the impact of scheduling a follow-up appointment prior to hospital discharge on 30-day readmission rates in patients with a primary diagnosis of HF. 


\section{Review of the Literature}

A search of the MEDLINE, CINAHL, Ovid and PubMed databases was performed using the terms "heart failure" and "readmission" and the linked terms "heart failure" and "readmission", "heart failure" and "transition" and "heart failure" and “follow-up" for the years 2006 through 2012. Next, an overview of HF will be presented followed by a review of the literature related to hospital readmission in general and hospital readmission in HF.

\section{Heart Failure}

HF is a chronic condition that occurs when the heart cannot pump enough blood and oxygen to the rest of the body (CDC, 2012). Certain conditions such as coronary heart disease, hypertension and diabetes can cause damage to the heart muscle, causing HF. Over time the heart continues to weaken and is not able to adequately fill and pump blood as well as it should (NIH, 2012). Approximately half of all people with HF will die within five years of initial diagnosis. There is no cure for HF but with treatment and early diagnosis, quality of life can be improved and mortality rates decreased.

Approximately 5.7 million people in the US are living with HF, costing the US 34.4 billion dollars each year (CDC).

In 2000 and 2010 there were approximately one million hospitalizations for HF in the US. The majority of these patients were over the age of 65 in 2000; in 2010, however, the number of people under 65 has increased from $23 \%$ in 2000 to $29 \%$ in 2010. Many patients, especially those under 65, are discharged to home (Hall, Levant, \& DeFrances, 2012). Given the chronic nature and high incidence of HF, hospital 
admissions and readmissions are quite common and quite costly. Thirty-day readmission rates for $\mathrm{HF}$ are higher than any other condition at $26.9 \%$, compared to the average 30 day readmission rate of $19.6 \%$ for all diagnoses (Jencks, Williams, \& Coleman, 2009).

\section{Hospital Readmission}

Coleman, Parry, Chalmers, and Min (2006) completed a randomized controlled trial to determine if a care transitions intervention, designed to encourage patients and caregivers to take on a more active role during care transitions, would decrease hospital readmission rates. This study had a sample size of 750 community-dwelling adults age 65 or older, admitted to the hospital with one of 11 identified conditions. Subjects in the intervention group received tools to promote cross-site communication, encouragement to take a more active role in their care and to assert their preferences and guidance from a transition coach. The intervention was built on four pillars: assistance with medication self-management; a patient-centered record owned and maintained by the patient; timely follow-up with primary or specialty care; and a list of "red flags" that indicated signs of a worsening condition and instructions on what to do.

Readmission rates were measured for both groups at 30,90 and 180 days. Coleman et al. found that patients in the intervention group had lower, statistically significant readmission rates at 30 days $(p=.048)$ and 90 days $(p=.04)$ than the control group. They also found that intervention patients had lower, statistically significant mean hospital costs $(p=.049)$. This valuable research suggests that providing coaching to chronically ill older patients during care transitions can help to decrease readmission rates. Despite costs associated with hiring additional staff to serve as transition coaches, 
this research suggests that the overall hospital cost may still be lower in patients who receive this intervention.

Jencks, Williams and Coleman (2009) examined patterns of rehospitalization and the relationship between rehospitalization and demographic characteristics of patients and hospitals. Medicare claims data were reviewed for a 15-month period between 2003 and 2005 for 11,855,702 Medicare beneficiaries who were discharged from the hospital. Jencks et al. found that $19.6 \%$ of discharged patients were readmitted within 30 days, $30 \%$ were readmitted within 60 days and 34\% were readmitted within 90 days. Of the patients who were readmitted within 30 days, 50.2\% did not have a charge submitted for a physician's office visit between the date of discharge and the date of readmission. Also of note, the researchers found that HF was the most common reason for readmission among all discharges. This research clearly illustrates the significance of hospital readmissions in those over age 65 (Medicare beneficiaries), especially among those with HF.

Kelly (2011) completed a comprehensive review of the literature in an attempt to identify trends in readmissions and practices that prevent readmissions in patients with chronic disease. Kelly's goal was to determine if best practice guidelines for preventing readmission exist based on a review of the literature. Kelly found that there was a lack of high-level research regarding interventions to decrease hospital readmission; however, several themes were identified including: patient empowerment and caregiver inclusion, bridging discharge process from hospital to the client's home; improving self-care capacities; and better client understanding of self-administration of medication. Higher- 
level evidence is needed as models that address the issue of readmissions continue to evolve. Next, an overview of the current literature related to HF and hospital readmission will be provided.

\section{Heart Failure and Hospital Readmission}

Anderson et al. (2006) performed a systematic review of the literature in an attempt to organize the variables associated with the hospital readmission of HF patients. Their goal was to organize the variables into a usable framework to be implemented in clinical practice. The authors reviewed 31 research reports that were performed between 1986 and 2004. A content analysis of the sample was performed and factors associated with HF readmissions were grouped into categories and classified into five domains. The

five domains identified were demographic, physiologic, psychosocial, patient functioning and resource utilization. The demographic domain included factors such as age, gender and marital status, while the physiologic domain encompassed biophysical health, comorbidities and polypharmacy. Mental and emotional health was classified as part of the psychosocial domain while activities of daily living were incorporated into the patient functioning domain. Lastly, the resource utilization domain included factors such as human, financial and social resources. This framework could serve as a foundation for healthcare providers caring for patients with HF across a variety of settings, including hospitalized patients with HF.

Roe-Prior (2007) performed a secondary analysis of data collected in an earlier study to determine if socio-demographic factors (age, gender, race, living situation, marital status, education and income) were related to post-discharge service use 
(rehospitalizations, emergency department visits and acute unscheduled physician office or clinic visits) for elders hospitalized with HF. Roe-Prior recruited a sample of 103 patients $(n=103)$ from two hospitals. Demographic and clinical data were collected from subjects during an in-person interview and subjects were then contacted by phone at two, six, and 12 weeks post-discharge. The researchers found that socio-demographic factors do play a role in predicting post-discharge service use, but none were predictive of HF readmissions. This research suggests that severity of illness may be more predictive of HF readmissions, but that socio-demographic factors should still be considered.

Sochalski et al. (2008) performed a non-experimental study where data that had been previously collected in 10 randomized controlled trials of HF care management programs was pooled and reanalyzed. The authors examined two outcome measures: hospital readmission and readmission days. They found that patients who were enrolled in programs that utilized multidisciplinary teams with in-person communication had more significant reductions in hospital readmissions and readmission days than patients who received routine care: $2.5 \%$ reduction $(p=<.001)$ in readmission and $5.7 \%$ reduction $(p=<.001)$ in readmission days. This important research suggests that HF patients who participate in multidisciplinary HF programs are less likely to be readmitted to the hospital than those who do not participate.

Kwok, Lee, Woo, Lee and Griffith (2008) performed a randomized controlled trial of a community nurse-supported discharge program for patients with HF. The aim of this study was to evaluate the effectiveness in preventing readmissions and improving functional status of older adults with $\mathrm{HF}$ as well as evaluating the cost-effectiveness of 
this program. Hospitalized patients $(\mathrm{n}=105)$ with chronic HF were randomly assigned to control and intervention groups. The intervention group received visits from the community nurse before discharge, within seven days of discharge, weekly for four weeks, and then monthly. Subjects in the control and intervention groups received follow-up at the same medical clinics; however, only the intervention subjects received visits from the community nurse. The primary outcome of this study was the rate of unplanned readmissions at six months. Kwok et al. found that the rates of readmission at six months were not significantly different between the control and intervention groups ( $57 \%$ vs. $46 \%$ in intervention subjects, $\mathrm{p}=.233$ ), though overall lower readmission rates were reported for subjects in the intervention group.

Annema, Luttik and Jaarsma (2009) performed a descriptive, qualitative study examining reasons for readmission in patients with HF. Researchers collected data on 173 readmissions from the perspective of patients, caregivers, cardiologists, and HF nurses. Patients and caregivers were asked to state in their own words what they thought the reason for readmission was and if they felt that the readmission could have been prevented. Cardiologists and HF nurses were asked if they felt the readmission could have prevented and how. Annema et al. found that patients, caregivers, cardiologists, and HF nurses believed that a large portion of readmissions (21-33\%) could have been prevented. They also found that $18 \%$ of caregivers thought that more follow-up visits would have helped prevent readmission and all respondents thought that adequate help from the general practitioner could have prevented readmission. This research suggests that more frequent follow-up post-discharge with the general practitioner could have 
prevented hospital readmission in patients with HF. This research also suggests that follow-up with an advanced practice nurse (APRN) such as a clinical nurse specialist (CNS) or nurse practitioner (NP), a cardiologist or a primary care physician could be effective in preventing HF readmissions.

Williams, Akroyd and Burke (2010) studied the effectiveness of a transitional care service for patients with HF. Williams et al. performed a quasi-experimental study that involved 97 subjects with a primary diagnosis of HF over a period of 18 weeks. A CNS in the hospital visited intervention subjects to help prepare them for discharge and to facilitate the transition to home. The researchers also administered a questionnaire to subjects in the intervention group about their experience. After discharge, intervention subjects visited the nurse-led clinic or were visited at home by a HF nurse. The researchers found that the number of readmissions was higher in the control group compared to the transitional care group (14\% vs. $8.5 \%$; NS). Overall, subjects in the control group reported being pleased with the experience. This study highlights the importance of providing guidance to HF patients during the transition from hospital to home in order to prevent readmissions.

Bueno et al. (2010) performed an observational study of 6,955,461 Medicare feefor-service hospitalizations for HF between 1993 and 2006. Outcome measures included length of hospital stay, inpatient and 30-day mortality rates, and 30-day readmission rates. The researchers found that between 1993 and 2006, mean length of stay decreased from 8.81 days to 6.33 days $(\mathrm{p}=<.001)$. They also found that inpatient mortality decreased from $8.5 \%$ to $4.3 \%$ and 30 -day mortality decreased from $12.8 \%$ to $10.7 \%$ 
$(p=<.001)$. Thirty-day readmission rates were the only measure to increase, from $17.2 \%$ to $20.1 \%(\mathrm{p}=<.001)$. The results of this observational study suggest that many advances have been made since 1993 in the care of HF patients as evidenced by decreased mortality rates. Length of hospital stay has also decreased dramatically while 30 -day readmission rates have increased significantly. This suggests that HF patients may be leaving the hospital too soon and that initiatives aimed at decreasing readmission rates have been ineffective during the time period studied.

Joynt, Oraz and Jha (2011) performed a retrospective cohort study that examined if hospitals with more experience in caring for patients with HF provided better, more efficient care. Subjects in this study were Medicare fee-for service patients with a primary discharge diagnosis of HF. Discharges $(n=1,029,497)$ were reviewed from 4,095 hospitals in the US. Medicare claims were used to examine the relationship between hospital case volume and quality, outcomes, and costs for patients with HF. Joynt et al. found that patients with HF who were discharged from hospitals with a higher volume of HF received higher-quality care and had better outcomes, but at a higher cost. Admission to a hospital with a higher case volume of HF patients was associated with lower mortality, lower readmission rates and higher costs. Based on their research, Joynt et al. suggested that quality of care for HF patients is better at hospitals that care for more patients with this diagnosis, yet the reason for higher associated costs should be explored.

Volz et al. (2011) studied the impact of psychosocial factors on prognosis in chronic HF patients. The researchers performed a prospective cohort study of 111 $(n=111)$ patients with chronic HF who had participated in an exercise based cardiac 
rehabilitation program. Questionnaires were used to assess baseline and follow-up psychological data and mortality, readmission, and health-related quality of life (QOL). After controlling for disease severity, Volz et al. found that none of the psychological variables (depression, anxiety, vital exhaustion, Type D personality and social support) were associated with increased mortality. They did find that severe anxiety might be predictive of cardiac-related readmission $(\mathrm{p}=0.06, \mathrm{NS})$. This study suggests that psychological variables, especially severe anxiety, should be considered when caring for patients with HF.

Kociol et al. (2011) completed a non-experimental retrospective study that examined if patient demographic characteristics and physician density were associated with early physician follow-up in hospitalized patients with HF. The researchers included 30,136 Medicare beneficiaries from 225 hospitals with a primary diagnosis of HF who were discharged to home. Kociol et al. found that patients who lived in areas of low physician density were less likely to receive early follow-up and that early physician follow-up was quite low (38\%). The authors also found that the likelihood of early follow up in women was $13 \%$ lower than men $(\mathrm{p}=<.001)$ and that black subjects were $16 \%$ less likely to receive early follow up compared to other races $(p=<.001)$. This research suggests that in order to increase compliance with early physician follow-up there is a need to increase the number of physicians or NPs in certain areas.

Schopfer, Whooley, and Stamos (2012) performed a non-experimental, retrospective study that examined whether or not compliance with performance measures impacted 30-day mortality and 30-day readmission rates in patients with HF. The four 
specific performance measures examined were: evaluation of left ventricular systolic function; administration of angiotensin-converting enzyme inhibitor I or angiotensinreceptor blocker for left ventricular systolic dysfunction; offering smoking cessation advice and counseling; and providing discharge instructions. Data from 3,655 hospitals in the US was analyzed. Schopfer et al. found that only evaluation of left ventricular function was associated with 30 -day mortality rates $(\mathrm{p}=.016)$. The researchers also noted that only two of the performance measures were associated with lower 30-day readmission rates: evaluation of left ventricular function $(\mathrm{p}=.018)$ and smoking cessation counseling $(\mathrm{p}=.018)$. These findings suggest that current performance measures may not be adequately assessing the quality of care for HF patients.

\section{Critique of the Literature}

While the current literature surrounding HF and hospital readmissions is abundant, further research is needed. Most studies have focused primarily on community-dwelling older adults. Many of the larger studies focused only on Medicare beneficiaries, so results cannot be generalized to the population as a whole. Randomized controlled trials have been performed, primarily in the area of multifaceted care transition programs. While the literature suggests that these programs are effective in reducing hospital readmissions, hospitals may be unwilling to adapt such models secondary to initial cost and lack of resources. One can certainly make the case that the cost of initiating these programs is far less than the cost of hospital readmissions. Given that CMS has implemented decreased reimbursement for hospital readmissions, hospitals are making every attempt to implement programs and initiatives aimed at preventing 
readmissions even if the programs and initiatives are not evidence based. Future research investigating simple, low-cost initiatives that may help to reduce HF readmissions is needed, including the use of APRNs in an expanded role. The purpose of this study was to determine the impact of scheduling a follow-up appointment prior to hospital discharge on 30-day readmission rates in patients with a primary diagnosis of HF.

Next, the theoretical framework used to guide this research will be presented and discussed. 


\section{Theoretical Framework}

Transitions Theory (Meleis, 2010) was utilized to guide the design and implementation of this research. The aim of this middle-range theory is to assist nurses in facilitating positive transitions for patients and families. Meleis' theoretical framework focuses on types and patterns of transitions, properties of transition experiences, facilitators and inhibitors of transition conditions, process indicators, outcome indicators and nursing therapeutics. While working with patients and families, nurses may encounter several types of transitions, including developmental, health and illness, situational, and organizational. According to Meleis et al., transitions are complex and multidimensional. Meleis further identified awareness, engagement, change and difference, time span and critical points and events as the essential properties of transitions.

Meleis stated that "the second most important part of transition theory is having an understanding of the transition experience itself, which is defined as the experience of losses and gains, changes and transformations, and a passage from one state to another" (p. 953). Changes in health and illness, acute or chronic diagnoses and going in or out of hospitals are examples of transitions that nurses may encounter while caring for patients. The transition experience is mediated by many factors such as what else might be occurring in the person's life and whether or not the person is going through multiple transitions.

The Transitions Theory (Meleis) was extremely useful in guiding this research for a variety of reasons. Patients with HF are likely experiencing multiple transitions at any 
given point, specifically transitions related to changes in health and illness and going in and out of the hospital. This research focused primarily on HF patients who were discharged from the hospital who had thus experienced transition from hospital to home. It is critical that nurses understand that these patients are experiencing transitions and are able to help facilitate this. Meleis identified critical points as a property that can either facilitate or inhibit transitions. Discharge from hospital to home is a critical point for the HF patient that ideally would facilitate the transition period. Physician or LIP follow-up after hospital discharge is a crucial step in the transition from hospital to home, therefore providing patients with a scheduled appointment should facilitate this transition.

Next, study methods will be presented. 


\section{Methodology}

\section{Purpose}

The purpose of this project was to determine the impact of scheduling a follow-up appointment prior to hospital discharge on 30-day readmission rates in patients with a primary diagnosis of $\mathrm{HF}$.

\section{Research Question}

The research question was: What is the impact of scheduling a follow-up appointment with a primary care provider (PCP) prior to hospital discharge on 30-day readmission rates in patients with a primary diagnosis of HF?

\section{Background}

The current practice at The Miriam Hospital is for follow-up appointments to be booked prior to hospital discharge with the patient's PCP within 10 days of hospital discharge. Appointments are not booked for patients who are discharged to skilled nursing facilities or acute rehabilitation facilities. All appointments are booked by unit secretaries and are then transcribed onto the patient's discharge instructions and onto an appointment card that is given to the patient. Although appointments are supposed to be booked for all patients discharged to home, this does not always occur for a variety of reasons.

\section{Design}

The design for this project was a retrospective chart audit using two groups: patients with a primary discharge diagnosis of HF with follow-up appointments booked prior to hospital discharge (Group 1) and patients with a primary discharge diagnosis of 
HF without follow-up appointment booked prior to hospital discharge (Group 2).

Follow-up appointments with primary care physicians as well as cardiologists were included.

\section{Sample and Participants}

A convenience sample was derived from a review of subjects' medical records. Inclusion criteria included a primary discharge diagnosis of HF and discharged to home or assisted living. Exclusion criteria included patients discharged to a skilled nursing facility or acute rehabilitation facility.

\section{Site}

This research took place at The Miriam Hospital in Providence, RI. The Miriam Hospital is a major teaching hospital for Brown University Medical School and is a 247bed acute care facility.

\section{Procedures}

IRB approval. The research proposal was shared with the Senior Vice President for Patient Care Services and Chief Nursing Officer at The Miriam Hospital who fully endorsed this project. The research proposal was submitted to the Lifespan Institutional Review Board (IRB) and the Rhode Island College IRB.

Measurement. The student researcher developed a data collection tool that was used to collect data (Appendix). This tool included gender, discharge home with services (yes or no), length of stay, follow-up appointment booked (yes or no), readmission within 30 days (yes or no), insurance (yes or no) and ejection fraction (EF). 
Data collection. The primary researcher completed all data collection, which was performed between November 15, 2012 and February 15, 2013. The primary researcher received an electronic copy of a list of all patients discharged from The Miriam Hospital during the months of June, July, August and September 2012 with a primary diagnosis of HF. This list was transferred to a password protected thumb drive that remained in a locked office at The Miriam Hospital. Only the primary researcher and principal investigator had the password for this thumb drive. This list included patient name, medical record number, admission date and discharge date.

The primary researcher reviewed the electronic medical records (EMRs) of patients who were identified as having a primary diagnosis of HF and were discharged during the months of June, July, August and September 2012. One hundred and forty EMRs were reviewed to obtain the desired sample of 60 subjects.

Data analysis. Basic descriptive statistics were used to examine the study variables. Percentage of readmissions within 30 days in each group was compared to determine if those with follow-up appointments booked had a lower rate of readmission. Differences between groups and relationships between variables were also examined. 


\section{Results}

The total sample included 60 subjects: Group 1 (without follow-up appointment) included 29 subjects, and Group 2 (with follow-up appointment) included 31.

Demographic characteristics are illustrated in Table 1.

Table 1

Demographics by Group

\begin{tabular}{|l|l|l|l|l|l|l|}
\hline & \# male & \# female & \# w/services & Mean LOS & Range LOS & Mean EF \\
\hline $\begin{array}{l}\text { Group 1 (n=29) } \\
\text { (no f/u appointment) }\end{array}$ & $\begin{array}{l}19 \\
(66 \%)\end{array}$ & $\begin{array}{l}10 \\
(34 \%)\end{array}$ & $18(62 \%)$ & 5.069 & $2-29$ & 37.931 \\
\hline $\begin{array}{l}\text { Group 2 (n=31) } \\
\text { (f/u appointment) }\end{array}$ & $\begin{array}{l}20 \\
(65 \%)\end{array}$ & $\begin{array}{l}11 \\
(35 \%)\end{array}$ & $18(58 \%)$ & 4.387 & $1-13$ & 39.067 \\
\hline
\end{tabular}

As can be seen, both groups included more males than females. The majority in both groups were discharged with services, and mean LOS were reasonably comparable. The mean ejection fraction (EF) was 37.931 and 39.067 for groups one and two respectively.

Thirty-day readmission rates between the two groups were compared (Table 2).

Table 2

30-Day Readmission by Group

\begin{tabular}{|l|l|l|}
\hline & \# 30-day readmission & \% 30-day readmission \\
\hline $\begin{array}{l}\text { Group 1 (n=29) } \\
\text { (no f/u appointment) }\end{array}$ & 9 & 31.03 \\
\hline $\begin{array}{l}\text { Group 2 (n=31) } \\
\text { (f/u appointment) }\end{array}$ & 7 & 22.58 \\
\hline
\end{tabular}


The rate of readmission was lower for Group 2 (22.58\%) compared to Group 1 $(31.03 \%)$. Although the readmission rate was lower in group two, the difference between the groups was not statistically significant.

Group 2 included subjects who had follow-up appointments with either a PCP or a cardiologist. Table 3 illustrates the follow-up appointments and the 30-day readmission rate by type of practitioner.

Table 3

30-Day Readmission: Follow-up with Cardiologist vs. PCP

\begin{tabular}{|l|l|l|}
\hline & \# 30-day readmission & $\%$ 30-day readmission \\
\hline $\begin{array}{l}\text { F/u appointment with } \\
\text { cardiologist }(\mathrm{n}=13)\end{array}$ & 1 & 7.69 \\
\hline $\begin{array}{l}\text { F/u appointment with PCP } \\
(\mathrm{n}=18)\end{array}$ & 6 & 33.33 \\
\hline
\end{tabular}

The 30-day readmission rate for those who had an appointment with a PCP was dramatically higher than those who followed up with a cardiologist $(33.33 \%$ vs. $7.69 \%)$. 


\section{Summary and Conclusions}

Heart failure (HF) is a chronic illness affecting nearly six million people in the United States. Due to the chronic nature of HF the costs of treatment and management continue to rise. In 2010 the US spent nearly 40 billion dollars treating HF (CDC, 2010), much of which was related to hospital admissions and readmissions for HF. Patients with HF can be readmitted for a variety of reasons, but $37.0 \%$ of readmissions are for HF itself (Jencks, Williams, \& Coleman, 2009). These findings indicate that HF readmissions are fairly predictable in patients who were initially admitted with HF. Given the recent emphasis on decreased reimbursement to hospitals for readmissions within 30 days of discharge there is a need to identify strategies that will reduce readmission rates and keep patients out of the hospital.

The current practice at The Miriam Hospital is for follow-up appointments to be booked with the patient's PCP within 10 days of hospital discharge. Appointments are booked for all patients except those who are discharged to skilled nursing facilities or acute rehabilitation facilities. Appointments are booked by unit secretaries and are then transcribed onto the patient's discharge instructions and onto an appointment card that is given to the patient. Although appointments are supposed to be booked for all patients

discharged to home, this does not always occur for a variety of reasons. The purpose of this project was to determine the impact of scheduling a follow-up appointment prior to hospital discharge on 30-day readmission rates in patients with a primary diagnosis of HF. 
A retrospective chart audit was performed using two groups: patients with a primary discharge diagnosis of HF with follow-up appointments booked with either a PCP or cardiologist prior to hospital discharge and patients with a primary diagnosis of HF without a follow-up appointment booked. A convenience sample of 60 subjects meeting inclusion criteria was obtained from a list of all patients with a primary diagnosis of HF who were discharged from the Miriam Hospital during June, July, August, and September 2012. Basic descriptive statistics were used to determine relationships between variables and differences between groups.

Group one (no follow-up appointment) consisted of 29 subjects and group two (follow-up appointment booked) consisted of 31 subjects. Group one (66\% males, 34\% females) and group two (65\% males, 35\% females) were similar in EF (mean EF 37.931 versus 39.067) and LOS (mean LOS 5.069 days versus 4.387 days). Subjects in group one had higher 30 -day readmission rates than those in group two (31.03\% versus $22.58 \%$, NS). Group two included patients who had follow-up appointments booked with either a PCP or cardiologist. Those who had a follow-up appointment booked with a cardiologist had lower 30-day readmission rates than those who followed-up with a PCP (7.69\% versus $33.33 \%$, NS).

These findings suggest that booking a follow-up appointment for HF patients with a cardiologist prior to hospital discharge may help to decrease 30 -day readmission rates. While a follow-up appointment with a PCP may also help to decrease 30-day readmission rates, the rates of readmission were significantly lower in those who had an appointment with a cardiologist. Booking a follow-up appointment with a patient's cardiologist prior 
to hospital discharge is a simple, low-cost strategy that could help to prevent hospital readmissions in HF patients. It was unclear why some patients had appointments booked with their cardiologist and some did not. Appointments with cardiologists are not booked by unit secretaries, therefore were likely booked by the cardiologists themselves.

Although lower readmission rates were seen in those HF patients with follow-up appointments booked prior to hospital discharge, the results of this study were not statistically significant. This is likely attributable to the relatively small sample size of 60 subjects. Patients who had follow-up appointments booked with cardiologists may have undergone some type of planned intervention such as cardiac catheterization or implantable cardioverter-defibrillator insertion.

The results of this research are limited by the relatively small sample size of 60 subjects. Subjects discharged to skilled nursing facilities were not included because follow-up appointments are not booked prior to hospital discharge for these patients. A significant number of HF patients, especially those with late stage HF, are discharged to skilled nursing facilities and experience frequent hospital readmissions. For subjects who had follow-up appointments booked, it was assumed that the appointment was kept and all subjects attended the scheduled appointment. Without contacting physician offices, there was no way to determine if this actually occurred. It remains unclear why some patients had follow-up appointments booked with cardiologists and some did not. No data was collected to determine if these patients underwent procedures or who booked these appointments. The only demographic variable identified was gender; no attempt 
was made to identify other demographic information. Variables such as age and ethnicity could have impacted study results.

The results suggest that The Miriam Hospital could benefit from booking followup appointments with patients' cardiologists prior to hospital discharge for all HF patients. Upon admission, patients are currently asked to give the name of their PCP so that this information can be entered into the EMR. This would be an opportune time to also obtain the name of the patients' cardiologist so that it will be readily available when the patient is discharged. Unit secretaries could book follow-up appointments with the patients' cardiologist along with the PCP for all HF admissions. If patients do not have a cardiologist they could be set up with one before leaving the hospital. For those patients with no health insurance, a referral could be made to the free cardiology clinic, with a follow-up appointment booked prior to hospital discharge. There could also be an opportunity for HF patients to have a follow-up appointment at a hospital based HF clinic. The clinic could be staffed by a combination of cardiologists and nurse practitioners (NPs). These suggestions will be shared with appropriate members of hospital leadership. 


\section{Recommendations and Implications}

Follow-up is paramount in preventing hospital readmissions for HF. While patients with HF should also follow-up with a PCP, follow-up with a specialist appears to be a key element of the discharge plan that may help to keep these patients out of the hospital. Coleman, Perry, Chalmers, and Min (2006) found that patients who followed up with a primary care or specialty physician after hospital discharge had lower, statistically significant readmission rates at 30,60 , and 90 days. The intervention subjects in this study also received assistance with medication self-management, a patient-centered record owned and maintained by the patient, and a list of "red flags" that indicated signs of a worsening condition and instructions on what to do. As illustrated by the results of this research and current literature, HF patients and hospitals can benefit from providing follow-up appointments with specialists.

Preventing HF readmissions is a key quality initiative. APRNs, specifically NPs, can play a vital role in preventing HF readmissions in a variety of ways. Acute care NPs who are caring for hospitalized HF patients should ensure that their patients have a follow-up appointment booked with either their cardiologist or a specialty HF clinic. NPs in the hospital also have a crucial role in preparing these patients for discharge. In collaboration with case managers and HF transition coaches, acute care NPs should provide HF patients with a thorough explanation of medications, signs and symptoms to watch for and the importance of timely follow-up during the discharge process. Acute care NPs can also play a role in providing specialized follow-up care for HF patients either in the setting of a cardiologist's office or in a HF clinic. 
Meleis et al. (2000) identified four types of transitions that patients experience: developmental; situational; health and illness; and organizational. HF patients will likely experience more than one of these transitions at a given time. During the hospital discharge process HF patients are experiencing health/illness transitions as well as organizational transitions. Each transition experience is unique and multidimensional and will vary based on the individual patient. Critical points and events such as acute illness and discharge from the hospital are key elements of the transition process and should be carefully considered for HF patients. Acute care NPs should pay close attention to these key elements in order to ensure that patients are safely transitioning from the hospital to home. Follow-up appointments should be booked and reviewed with patients prior to transitioning home to ensure that timely follow-up occurs.

Follow-up appointments with cardiologists or specialty HF clinics should be considered standard of care for all HF patients admitted to the hospital. Those patients discharged to skilled nursing facilities should also have scheduled follow-up with a cardiologist or NP given the high overall rate of hospital readmission for HF patients. Jencks, Williams, and Coleman (2009) found that in $50.2 \%$ of patients who were readmitted to the hospital within 30 days of discharge there was no evidence that a physician follow-up occurred. Although this study was not limited to HF, Jencks et al. found that HF had the highest 30-day readmission rate among Medicare beneficiaries for all medical and surgical diagnoses.

Further research is warranted using a larger sample size at multiple sites. An attempt should be made to include patients discharged to skilled nursing facilities in 
future research as these patients account for a large number of HF patients. Research should also be done to specifically examine follow-up appointments with cardiologists. Data should be collected about any invasive procedures that took place during hospitalization, such as implantable cardioverter-defibrillator insertion, to determine if there is any correlation between invasive procedures and hospital readmission rates. Institutions should consider including booking follow-up appointments as part of a standardized discharge plan for all HF patients. Many hospitals have created HF "bundles" that incorporate multiple evidence-based interventions aimed at preventing hospital readmissions. APRNs can be key in developing, implementing, and evaluating comprehensive strategies to reduce HF readmissions. These bundles should include booking follow-up appointments prior to hospital discharge with a cardiologist or NP at a specialty HF clinic. Given the current emphasis on quality and the importance of preventing hospital readmissions, HF "bundles" should not only be standard of care but should be incorporated into revised hospital policies. Compliance with these evidencebased interventions should no longer be optional or suggested as hospitals are losing money due to decreased reimbursement for hospital readmissions. APRNs have a key role in advocating for policy changes at the state and national level that promote health of the public and reduce the negative consequences associated with hospital admission. NPs need to continue to be actively involved in inter-disciplinary insititutional, state, and national efforts to improve the care of individuals with HF. 


\section{References}

Andreson, M. A., Levsen, J., Dusio, M. E., Bryant, P. J., Brown, S. M., Burr, C. M., \& Brown-Benn, C. (2006). Evidenced-based factors in readmission of patients with heart failure. Journal of Nursing Quality, 21(2), 160-167.

Annema, C., Luttik, M. L., \& Jaarrsma, T. (2009). Reasons for readmission in heart failure: perspectives of patients, caregivers, cardiologists, and heart failure nurses. Heart \& Lung, 38(5), 427-434.

Bueno, H., Ross, J. S., Wang, Y., Chen, J., Vidan, M. T., Normand, S. T., ... Krumholz, H. M. (2010). Trends in length of stay and short-tern outcomes among medicare patients hospitalized for heart failure, 1993-1006. JAMA, 303(21), 2141-2147.

Center for Disease Control and Prevention. (2012, October). Heart failure fact sheet. Retrieved from http://www.cdc.gov/DHDSP/data_statistics/fact_sheets/fs_heart_failure.htm

Coleman, E. A., Parry, C., Chalmers, S., \& Min, S. (2006). The care transitions intervention: results of a randomized controlled trial. Archives of Internal Medicine, 166, 1822-1828.

Hall, M. J., Levant, S., \& DeFrances, C. J. (2012). Hospitalizations for congestive heart failure: United States, 2000-2010. NCHS Data Brief, 108, 1-8. Retrieved from http://www.cdc.gov/nchs/data/databriefs/db108.pdf.

Jencks, S. F., Williams, M. V., Coleman, E. A. (2009). Rehospitalizations among patients in the Medicare fee-for-service program. The New England Journal of Medicine, 360(14), 1418-1428. 
Joynt, K. E., Orav, E. J., \& Jha, A. K. (2011). The association between hospital volume and processes, outcomes and costs of care for congestive heart failure. Annals of Internal Medicine, 154(2), 94-102.

Kelly, M. (2011). Self management of chronic disease and hospital readmission: a care transition strategy. Journal of Healthcare of Chronic Illness, 3, 4-11.

Kociol, R. D., Greiner, M. A., Fonarow, G. C., Hammill, B. G., Heidenreich, P. A., Yancy, C. W., Peterson, E. D., Curtis, L. H., \& Hernandez, A. F. (2011). Associations of patient demographic characteristics and regional physician density with early physician follow-up among Medicare beneficiaries hospitalized with heart failure. The American Journal of Cardiology, 108, 985-991.

Kwok, T., Lee, J., Woo, J., Lee, D. T., \& Griffith, S. (2008). A randomized controlled trial of a community nurse-supported hospital discharge programme in older patients with chronic heart failure. Journal of Clinical Nursing, 17, 109-117.

Meleis, A. I. (2010). Epilogue (frequently asked questions). In A. I. Meleis (Ed.), Transitions theory: middle-range and situation-specific theories in nursing research and practice (952-961). New York, NY: Springer.

Meleis, A. I., Sawyer, L. M., Im, E., Messias, D. K. H., \& Schumacher, K. (2010). Experiencing transitions: an emerging middle-range theory. In A. I. Meleis (Ed.), Transitions theory: middle-range and situation-specific theories in nursing research and practice (111-130). New York, NY: Springer.

National Heart and Lung Institute. (2012, January). Explore heart failure. Retrieved from http://www.nhlbi.nih.gov/health/health-topics/topics/hf/. 
Roe-Prior, P. (2007). Sociodemographic variables predicting poor post-discharge outcomes for hospitalized elders with heart failure. MEDSURG Nursing, 16(5), 317-321.

Schopfer, D. W., Whooley, M. A., Stamos, T. D. (2012). Hospital compliance with performance measures and 30-day outcomes in patients with heart failure. American Heart Journal, 164(1), 80-86.

Sochalski, J., Jaarsma, T., Krumholz, H. M., Laramee, A., McMurray, J. J. V., Naylor, M. D., Rich, M. W., Riegel, B., \& Stewart, S. (2009). What works in chronic care management: the case of heart failure. Health Affairs, 28(1), 179-189.

Stone, J. \& Hoffman, G. J. (2010, September 21). Medicare hospital readmissions: issues, policy options and PPACA. Retrieved from http://www.ncsl.org/documents/health/Medicare_Hospital_Readmissions_and_PP ACA.pdf

Volz, A., Schmid, J., Zwahlen, M., Kohls, S., Saner, H., \& Barth, J. (2011). Predictors of readmission and health related quality of life in patients with chronic heart failure: a comparison of different psychosocial aspects. Journal of Behavioral Medicine, 34, 13-22.

Williams, G., Akroyd, K. \& Burke, L. (2010). Evaluation of the transitional care model in chronic heart failure. British Journal of Nursing, 19(22), 1402-1407. 


\section{Appendix}

Data Collection Tool

\begin{tabular}{|c|c|c|c|c|c|c|c|}
\hline$\#$ & Gender & Services & $\begin{array}{c}\text { Length } \\
\text { of Stay }\end{array}$ & $\begin{array}{c}\text { Follow-up } \\
\text { Appointment }\end{array}$ & $\begin{array}{c}\text { 30-day } \\
\text { Readmission }\end{array}$ & Insurance & $\begin{array}{c}\text { Ejection } \\
\text { Fraction }\end{array}$ \\
\hline & & & & & & & \\
\hline & & & & & & & \\
\hline
\end{tabular}

